\title{
Atom-by-atom and concerted hopping of adatom pairs on an open metal surface
}

\author{
A. Bogicevic, ${ }^{a}$ S. Ovesson, ${ }^{b}$ B. I. Lundqvist, ${ }^{b}$ and D. R. Jennison ${ }^{a}$ \\ ${ }^{a}$ Surface and Interface Sciences Department, Sandia National Laboratories, Albuquerque, NM 87185-1429, USA \\ ${ }^{b}$ Department of Applied Physics, Chalmers University of Technology and Göteborg University, S-41296 Göteborg, Sweden
}

(August 4, 1999)

Atom-by-atom and concerted hopping of ad-dimers on the open (100) surface of fcc metals are studied by means of density-functional calculations. The adatom interaction is relatively short-ranged, and beyond next-nearest neighbors ad-dimers are effectively dissociated. Diffusion takes place by a simple shearing process, favored because it maximizes adatom coordination at the transition stat $\mathrm{S}_{\mathrm{s}}$ This holds for $\mathrm{Al}, \mathrm{Au}$, and $\mathrm{Rh}$, and is likely a general result because geometrical arguments dominate over details of the electronic structure.

PACS number(s): 68.35.Fx, 68.35.Bs, 68.35.Jk

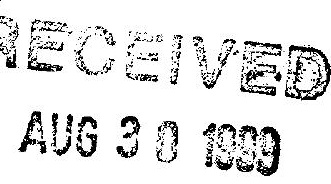

The continuous drive to produce smaller and faster electronic components has led to radical changes in manufacturing methods. For example, the transistors and diodes in modern microchips, laserpens, and other electronic gadgets, all contain minute junctions that have been grown essentially atom by atom. There is thus a great technological incentive to study the interactions between deposited atoms, which has lead to substantial experimental and theoretical efforts [1-3].

A comprehensive picture, connecting microscopic binding and kinetic barriers with meso- and macroscopic growth modes and patterns, requires insight into the motion and aggregation of ad-particles $[1,2]$. Whereas numerous experiments and accurate theoretical calculations have addressed the diffusion of single adatoms [1-4], few studies have dealt with the properties of pairs of adatoms. Ad-dimers nucleate larger islands, strongly influencing early stages of growth, and can also induce morphological roughening [2]. Still, the mechanism by which they diffuse and dissociate on the open (100) surface of fcc metals is largely unknown, which is a serious problem since this is one of the most commonly exposed crystal faces.

Insight into the cohesion and migration of ad-dimers is not just needed for realistic modelling, but also essential to interpret experimental data. Aside from a few materials amenable to field-ion microscopy (FIM) study, the primary means of measuring single atom diffusivities is to rely on nucleation theory to interpret island density data obtained with scanning-tunneling microscopy $[1,2]$. A potential problem with this method is that since dimers affect the island density, the correct monomer diffusivity can only be extracted if dimer stability and mobility are correctly accounted for in the nucleation formulas. Thus, without information about dimer properties, the door is open for misinterpretations of experimental data.

A question of intrinsic interest concerns the mobility of ad-dimers versus adatoms. On close-packed surfaces, dimer migration has in some systems been predicted to be greatly enhanced by concerted diffusion [5,6]. Still, adatoms are typically more mobile than ad-dimers on the (111) face of metals [1,2]. On open surfaces, the results vary, and there is no such general trend [1]. Early accounts of enhanced ad-dimer mobility due to rebonding [7] have recently been contradicted for a variety of metal systems [8], and it thus remains to find an explanation for these variations.

In this Letter, we reveal the mechanisms of diffusion and dissociation for atom-by-atom and concerted hopping of ad-dimers on an open (100) metal surface. Results for $\mathrm{Al}_{2} / \mathrm{Al}(100), \mathrm{Au}_{2} / \mathrm{Au}(100)$, and $\mathrm{Rh}_{2} / \mathrm{Rh}(100)$ are qualitatively identical, and likely to carry over to other metals as well since geometrical aspects are found to dominate over details of the electronic structure. The relation between adatom and ad-dimer mobility is determined and explained in terms of transition-state coordination. Over a dozen computed activation energies are rationalized in a pair-wise model based on two fundamental quantities, which should greatly facilitate parametrization in kinetic simulations.

The calculations are based on density-functional theory (DFT) $[9,10]$, using a pseudopotential method, as implemented in the VASP [11] and dacapo [12] codes. For the exchange-correlation functional, the generalized gradient approximation (GGA) [13] is used for $\mathrm{Al}$ and $\mathrm{Rh}$, and the local-density approximation (LDA) [14] for Au. Note that, unlike in the perturbative [15] "post-LDA/GGA" approach, these calculations are all fully self-consistent. The one-electron wave functions are expanded in a planewave basis with an energy cutoff of $9,13,15 \mathrm{Ry}$ for $\mathrm{Al}$, $\mathrm{Au}, \mathrm{Rh}$, respectively, using ultra-soft Vanderbilt pseudopotentials [16]. The Kohn-Sham equations are solved iteratively, and the atomic structure is optimized until the forces on all unconstrained atoms are less than 0.03 $\mathrm{eV} / \AA$. The (100) super-cell is constructed of 8 layers each containing 20 atoms. Above an additional adsorbate layer [17], there is more than $10 \AA$ of vacuum. The Brillouin zone is sampled using a dense $6 \times 6(4 \times 4) \mathbf{k}$ point mesh for $\mathrm{Al}$ ( $\mathrm{Au}$ and $\mathrm{Rh}$ ) [18]. Transition states are located by mapping out the total energy on a mesh centered at geometric symmetry (bridge) sites. At each 


\section{DISCLAIMER}

This report was prepared as an account of work sponsored by an agency of the United States Government. Neither the United States Government nor any agency thereof, nor any of their employees, make any warranty, express or implied, or assumes any legal liability or responsibility for the accuracy, completeness, or usefulness of any information, apparatus, product, or process disclosed, or represents that its use would not infringe privately owned rights. Reference herein to any specific commercial product, process, or service by trade name, trademark, manufacturer, or otherwise does not necessarily constitute or imply its endorsement, recommendation, or favoring by the United States Government or any agency thereof. The views and opinions of authors expressed herein do not necessarily state or reflect those of the United States Government or any agency thereof. 


\section{DISCLAIMER}

Portions of this document may be illegible in electronic image products. Images are produced from the best available original document. 

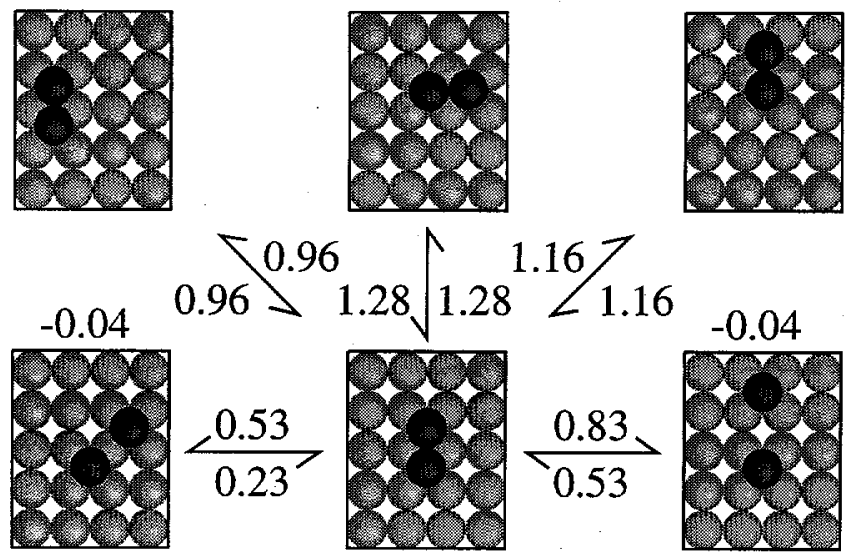

\section{$\lceil 0.96$}

$0.96 \unlhd 1.28 \sqrt{1.28} / 1.16 / 16$

\section{$<\frac{0.53}{0.23}$}
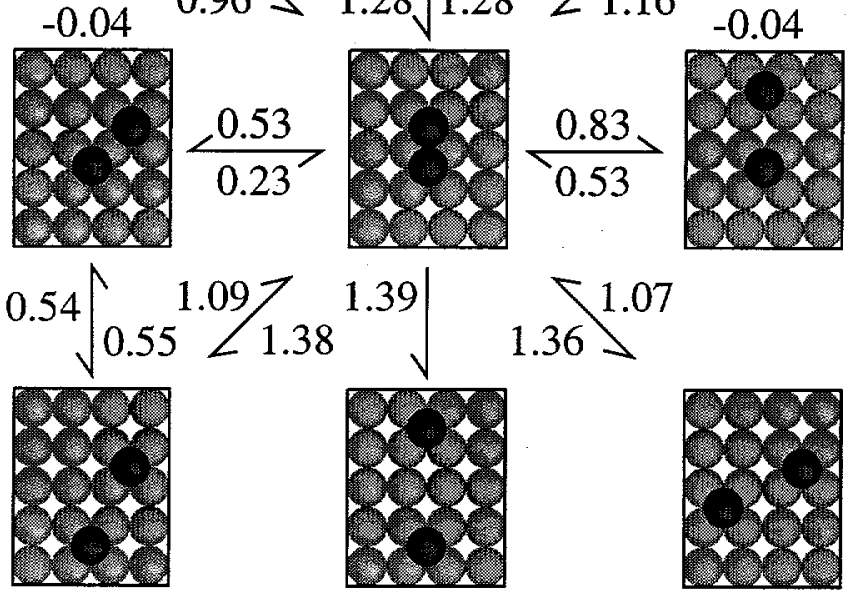

$-0.03$
$1.36 \mathrm{D}$

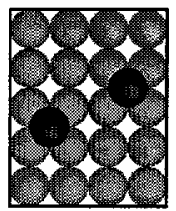

$-0.03$

FIG. 1. Activation energies of diffusion and dissociation processes for $\mathrm{Al}_{2} / \mathrm{Al}(100)$ calculated within DFT-GGA. All values are in $\mathrm{eV}$. The barriers depend on the direction of the motion, as indicated with arrows. Binding energies of metastable sites (with respect to isolated adatoms) are noted at the top or bottom of the individual configurations.

point, vertical relaxation is allowed, while one or two of the lateral degrees of freedom are frozen for the adparticle(s). Typically, the true saddle-point is shifted away from the symmetry site by a few tenths of an $\AA$.

Since diffusion and dissociation of ad-dimers on the open (100) face of fcc metals are almost completely unknown, many complex paths need be considered. To this end, we map out a large variety of hopping barriers for $\mathrm{Al}_{2} / \mathrm{Al}(100)$, both probable and improbable, thereby isolating the most facile processes. We then study these also for the $\mathrm{Au}_{2} / \mathrm{Au}(100)$ and $\mathrm{Rh}_{2} / \mathrm{Rh}(100)$ systems, and finally compare and analyze the results in detail.

Two adatoms occupying neighboring hollows form the ground state of the ad-dimer. The GGA (LDA) ad-dimer binding energy of the equilibrium configuration, defined with respect to two isolated adatoms, is $E_{b}=0.26,0.18$, and $0.39 \mathrm{eV}(0.30,0.27,0.54 \mathrm{eV})$ for $\mathrm{Al}, \mathrm{Au}$, and $\mathrm{Rh}$, respectively [8]. Unlike on close-packed surfaces [5,6], adatom interactions are very short-ranged, and beyond next-nearest neighbors ad-dimers are effectively dissociated, as discussed in detail below.

At this point, we focus on $\mathrm{Al}$ to isolate the most facile hopping processes. The activation energies for a variety of atom-by-atom and concerted hopping events are shown in Fig. 1. We find that these barriers can be rationalized in a simple manner: Assuming for a moment that both

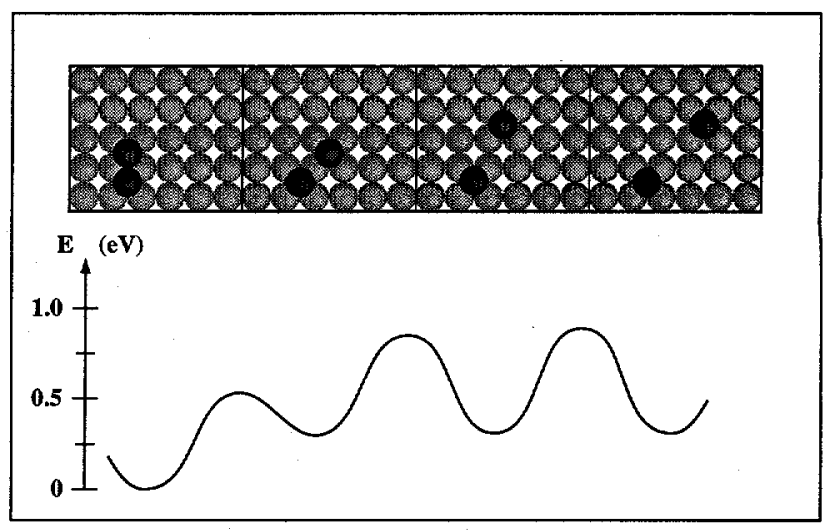

FIG. 2. Schematic illustration of dimer dissociation by hopping for $\mathrm{Al}_{2} / \mathrm{Al}(100)$. Note that the next-nearest neighbor configuration has a relatively shallow adsorption well, which effectively increases the dissociation barrier.

electronic and elastic adatom interactions at transition states are negligible, the activation energy $\Delta E$ for any diffusion/dissociation event can be related to the monomer diffusion barrier $E_{1}=0.58 \mathrm{eV}$ and ad-dimer binding energy $E_{\mathrm{b}}=0.26 \mathrm{eV}$ according to

$$
\Delta E=n E_{1}+m E_{\mathrm{b}}
$$

where $n$ denotes the number of saddle-points crossed from one configuration to another, and $m=1$ if the adatoms are separated beyond nearest neighbors, and $m=0$ otherwise. Comparisons with the actual DFT barriers (Fig. 1) show that this simple formula holds to within $0.1 \mathrm{eV}$ for all processes except concerted sliding perpendicular to the dimer axis (center to up/left-most box in Fig. 1) and dimer shearing (middle row, center to left). The accuracy of this expression increases with the transition-state adatom separation because of weaker electronic interaction (Fig. 1), and beyond a separation of $\approx 4 \AA$, deviations from Eq. 1 are very small and mainly due to elastic interactions via the substrate [8]. It is of course possible to adjust the parameters appearing in Eq. 1, so as to obtain a closer fit to the calculated barriers, which might be useful in kinetic simulations.

At first glance, the activation energies depicted in Fig. 1 imply that the ad-dimer dissociates by shearing with a barrier as low as $0.53 \mathrm{eV}$. However, the sheared dimer is in a metastable state, and the barrier to restore the ground-state configuration is much smaller $(0.23 \mathrm{eV})$ than the shearing barrier itself, see Fig. 2. Consequently, the likelihood of further separation is greatly diminished. As pointed out by Bartelt and coworkers [21], the effective barrier for separation is independent of the dissociation path, as long as no individual process along the path has a barrier larger than $E_{1}+E_{\mathrm{b}}$. Thus, dissociation of $\mathrm{Al}$ dimers on $\mathrm{Al}(100)$ by hopping proceeds via bond breaking both parallel and perpendicular to the dimer axis (but not through any collective process), with an ef- 
fective dissociation barrier close to $E_{1}+E_{\mathrm{b}}=0.84 \mathrm{eV}$, as illustrated in Fig. 2.

As revealed in Fig. 1, ad-dimer diffusion proceeds by shearing and subsequent recombination, a two-step process wherein both the orientation and center of mass of the dimer may or may not change depending on the relative directions of the two jumps. Diffusion by stretching (middle row, center to right in Fig. 1) and recombination, concerted sliding, or concerted rotation is considerably less facile. This is easy to understand by considering the transition-state coordination, suggesting a general validity for these results, as discussed in detail below.

Enlightened with the results for $\mathrm{Al}$, we proceed to study the most facile processes (i.e. shearing and stretching) for $\mathrm{Au}_{2} / \mathrm{Au}(100)$ and $R h_{2} / \mathrm{Rh}(100)$. As expected, diffusion by shearing is strongly favored over stretching for these two systems as well. The barrier for shearing in the three systems studied is $0.2-0.3 \mathrm{eV}$ smaller than for stretching (Table I), meaning that diffusion by stretching is rare even at elevated substrate temperatures. This is consistent with FIM studies of $\mathrm{Rh}_{2} / \mathrm{Rh}(100)$, where the dimer-axis orientation is observed to change during diffusion (at temperatures where dimers are stable), which is not possible if stretching alone prevailed.

There is a simple and transparent way of rationalizing the dissociation and diffusion paths in terms of transition-state coordination. Ad-dimers have fewer neighbors than bulk atoms, so the preservation of a short adatom-adatom bond increases the binding in equilibrium configurations and decreases the barrier at saddlepoints. Shearing is thus favored over stretching because the adatom-adatom bond-length at the transition state is $52-59 \%$ longer in the latter case $\left[r_{\mathrm{aa}}^{\mathrm{st}} / r_{\mathrm{aa}}^{\mathrm{sh}}=4.32 / 2.72\right.$, $4.40 / 2.89,4.10 / 2.66 \AA$ for $\mathrm{Al}, \mathrm{Au}$ and $\mathrm{Rh}$, respectively], and the corresponding barrier therefore substantially larger. Variations in adatom-substrate bond lengths are discussed below in conjunction with rebonding effects.

TABLE I. Diffusion barriers for ad-dimer shearing $\left(E_{2}^{\mathrm{sh}}\right)$ and ad-dimer stretching $\left(E_{2}^{\text {st }}\right)$ in $\mathrm{eV}$, and bond lengths for shearing in $\AA: r_{\text {as }}$ denotes the average bond length between the adatom left behind in the process and the four underlying nearest neighbors, $r_{\text {aa }}$ the adatom-adatom bond length. The values are given at equlibrium binding (Eq.) and transition state (TS); $\mathrm{TS}^{*}$ denotes the adatom-adatom bond length if the adatom left behind is not allowed to relax towards the adatom diffusing away. The parameter $\delta_{1}$ yields the difference between Eq. and TS bond-length values, showing that there is no rebonding; $\delta_{2}$ gives the difference between TS and TS* values, showing the importance of lateral coordination.

\begin{tabular}{c|cc|ccccccc}
\hline \hline & & & \multicolumn{4}{|c}{$r_{\mathrm{as}}$} & \multicolumn{5}{c}{$r_{\mathrm{aa}}$} \\
& $E_{2}^{\text {sh }}$ & $E_{2}^{\text {st }}$ & Eq. & TS & $\delta_{1}$ & Eq. & TS & $\mathrm{TS}^{*}$ & $\delta_{2}$ \\
$\mathrm{Al}$ & 0.53 & 0.83 & 2.74 & 2.73 & 0.00 & 2.66 & 2.72 & 2.87 & 0.15 \\
$\mathrm{Au}$ & 0.86 & 1.11 & 2.71 & 2.72 & 0.01 & 2.76 & 2.89 & 3.13 & 0.23 \\
$\mathrm{Rh}$ & 1.07 & 1.27 & 2.57 & 2.60 & 0.03 & 2.64 & 2.66 & 2.88 & 0.22 \\
\hline \hline
\end{tabular}

Concerted processes are found to be rather unfavorable for similar reasons. Concerted dissociation (bottom row of Fig. 1) is very costly because it requires the passing of two bridge sites simultaneously, and both adatoms are severely under-coordinated (far apart) at the transition state. Diffusion by concerted sliding, found to be the dominant process for $\mathrm{Al}_{2} / \mathrm{Al}(111)$ [5] and $\mathrm{Pt}_{2} / \mathrm{Pt}(111)[6]$, is unfavored here because the energy gain in retaining a short ad-dimer bond is smaller than the energy cost of simultaneously breaking four bonds by displacing two adatoms from their equilibrium binding sites to saddle-points (near bridge sites). The latter energy is considerably smaller on the close-packed (111) surface [1] (partly due to the loss of only 2 bonds), which is why on this surface the balance can be reversed.

Armed with new insight into ad-dimer diffusion, we return to the issue of adatom versus ad-dimer mobility. The respective diffusion barriers are $E_{2}^{\text {sh }} / E_{1}=0.58 / 0.53$, $0.84 / 0.86$ and $0.89 / 1.07 \mathrm{eV}$ for $\mathrm{Al}, \mathrm{Au}$, and $\mathrm{Rh}$, respectively, where $E_{2}^{\mathrm{sh}}\left(E_{1}\right)$ denotes the activation energy for ad-dimer shearing (adatom diffusion). Previous theoretical calculations for $E_{1}$ yield $0.83 \mathrm{eV}$ for $\mathrm{Au}$ (LDA) [22] and $0.89 \mathrm{eV}$ for Rh (GGA) [4], and the most accurate experimental value for $\mathrm{Rh}$ is $0.88 \mathrm{eV}$ [23] - all in excellent agreement with the results presented here. The diffusion of single $\mathrm{Al}$ atoms on $\mathrm{Al}(100)$ has been subject to numerous theoretical studies $[4,7,20]$, and our $0.58 \mathrm{eV}$ is somewhat higher than the most recently reported GGA value of $0.51 \mathrm{eV}$ [4] (obtained using a substantially smaller unit-cell) [24]. The FIM result for the $\mathrm{Rh}_{2} / \mathrm{Rh}(100)$ diffusion barrier is $0.97 \mathrm{eV}$ (assuming a prefactor $D_{0}$ of $10^{-} 3 \mathrm{~cm}^{2} / \mathrm{s}$ ) [25], somewhat smaller than our $1.07 \mathrm{eV}$ (which when corrected for zero-point vibrations is likely to drop to about $1.04 \mathrm{eV}$ [5]).

The diffusivities of monomers and dimers are apparently relatively similar, which is unexpected in view of rebonding theory [7], wherein ad-dimers should be more mobile than adatoms because of longer ad-surface bonds and a relaxation process in which the atom not surmounting the barrier strengthens its bond to the surface as its partner moves away. Neither of the two rebonding theory arguments for augmented ad-dimer mobility are borne out in the three systems studied here: Ad-dimers do not reside further above the surface than adatoms (in a presumably less corrugated potential), and the remaining adatom actually relaxes towards the diffusing adatom, slightly weakening its bonds to the surface, (for $\mathrm{Al}$, the average bond length to the surface is unchanged), see Table $\mathrm{I}$. The relaxation of the remaining adatom is mainly lateral, and in the case of $\mathrm{Al}$ lowers the barrier for shearing by about $0.06 \mathrm{eV}$. Similar results for a variety of other metal systems have been reported recently [8].

Finally, one might wonder how transferrable these results are to other (100) fcc metal systems. We argue here that there are several reasons to believe that the results and analysis presented here are quite general. First 
of all, the results are dominated by structure: the open (100) surface offers a high adatom coordination with deep adsorption hollows, which is why both adsorption energies and self-diffusion barriers for adatoms as a rule are much larger on open surfaces than on close-packed ones [26]. For this reason, many-body subtleties of the atomic interaction are less important, and screening effectively isolates atoms from each other even at very short separations. Moreover, the weak adatom interaction beyond next-nearest neighbors $(\lesssim 40 \mathrm{meV}$ for $\mathrm{Al} / \mathrm{Al}(100))$ is predominantly elastic [8], and thus indirect and largely undirectional (the latter point was first made by Feibelman [7]). Adatoms on an open metal surface therefore quite generally behave independently beyond small separations. This qualitative agreement between simple, noble, and transition metals is an attractive feature, evident also in dimer studies on close-packed surfaces $\left(\mathrm{Al}_{2} / \mathrm{Al}(111)\right.$ [5] and $\mathrm{Pt}_{2} / \mathrm{Pt}(111)$ [6]). There is, however, more work to be done here, since in some systems ad-dimers may diffuse by exchange, i.e. concerted substitution with substrate atoms $[4,19]$, (so far inferred experimentally only for $\mathrm{Pt}_{2} / \mathrm{Pt}(100)$ [19] with help from semi-empirical calculations).

In summary, we provide a detailed first-principles mapping of ad-dimer diffusion and dissociation by hopping on the open (100) surface of fcc metals. We show that diffusion proceeds by shearing because it maximizes adatom coordination at the saddle-point. This explanation implies that shearing should be the preferrable diffusion path for ad-dimers on any fcc (100) metal surface devoid of exchange processes. We further show that even complicated collective processes can be accounted for to good accuracy with a simple pair-wise model based on the adatom diffusion barrier and the ad-dimer binding energy, which should greatly facilitate parametrization in kinetic Monte Carlo simulations. Rebonding arguments are found to be invalid, and a new and simple picture is developed in terms of transition-state coordination.

We thank Brian Swartzentruber for a critical reading of the manuscript and Gary Kellogg for constructive remarks. VASP was developed at the Institut für Theoretische Physik of the Technische Universität Wien. Sandia is a multiprogram laboratory operated by Sandia Corporation, a Lockheed Martin Company, for the United States Department of Energy under Contract DE-AC0494AL85000. This work was partially supported by a Laboratory Directed Research and Development project and by the Swedish Research Counsil for Engineering Sciences (TFR). Allocation of computer time at the UNICC facilities at Chalmers University of Technology is gratefully acknowledged.
[1] G. Kellogg, Surf. Sci. Rep. 21, 1 (1994).

[2] H. Brune, Surf. Sci. Rep. 31, 121 (1998).

[3] C. Ratsch, P. Ruggerone, and M. Scheffler, in Surface Diffusion: Atomistic and Collective Processes, ed. by M. C. Tringides (NATO ASI Series B: Physics Vol. 360, Plenum Press, New York 1997).

[4] For a compilation of self-diffusion barriers on open metal surfaces, see P. J. Feibelman, Surf. Sci. 423, 169 (1999).

[5] A. Bogicevic, P. Hyldgaard, G. Wahnström, and B. I. Lundqvist, Phys. Rev. Lett. 81, 172 (1998).

[6] G. Boisvert and L. J. Lewis, Phys. Rev. B 59, 9846 (1999).

[7] P. J. Feibelman, Phys. Rev. Lett. 58, 2766 (1987).

[8] A. Bogicevic, Phys. Rev. Lett. 82, 5301 (1999).

[9] P. Hohenberg and W. Kohn, Phys. Rev. 136, B864 (1964).

[10] W. Kohn and L. J. Sham, Phys. Rev. 140, A1133 (1965).

[11] G. Kresse and J. Hafner, Phys. Rev. B 47, 558 (1993); 49, 14251 (1994); 54, 11169 (1996).

[12] B. Hammer et al., computer code dacapo-1.30, CAMP, Denmark Technical University.

[13] J. P. Perdew et al., Phys. Rev. B, 46, 6671 (1992).

[14] J. P. Perdew and A. Zunger, Phys. Rev. B, 23, 5048 (1981).

[15] M. Fuchs et al., Phys. Rev. B 57, 2134 (1998).

[16] D. Vanderbilt, Phys. Rev. B 32, 8412 (1985).

[17] The artificial electric field induced by one-sided adsorption is very weak; when compensated by a dipole layer in the vacuum, energy differences change by $\lesssim 10 \mathrm{meV}$.

[18] Use of a smaller mesh (Al) or larger mesh (Au and $\mathrm{Rh}$ ) changes $E_{b}$ by $\lesssim 5 \mathrm{meV}$; see also Ref [8].

[19] G. L. Kellogg and A. F. Voter, Phys. Rev. Lett. 67, 622 (1991);

[20] P. J. Feibelman, Phys. Rev. Lett. 65, 729 (1990); R. Stumpf and P. J. Feibelman, Phys. Rev. B 53, 4958 (1996).

[21] M. C. Bartelt, L. S. Perkins, and J. W. Evans, Surf. Sci. 344, L1193 (1995).

[22] B. D. Yu and M. Scheffler, Phys. Rev. B 56, 15569 (1997).

[23] G. Ayrault and G. Ehrlich, J. Chem. Phys. 60, 281 (1974).

[24] Our LDA value for this barrier is $0.61 \mathrm{eV}$, in good agreement with the GGA number; the LDA-GGA discrepancy is more severe in the case of $\mathrm{Au}$, where GGA yields a barrier of $0.58 \mathrm{eV}$.

[25] G. L. Kellogg, Phys. Rev. Lett. 73, 1833 (1990).

[26] For simple and noble metals like $\mathrm{Al}$ and $\mathrm{Cu}, E_{1}$ varies by a factor of $\simeq 20$ between the two crystal faces $[1,5,8,27]$; for transition metals, the variations are smaller $[1,8]$.

[27] A. Bogicevic, S. Ovesson, B. I. Lundqvist, and D. R. Jennison, to be published. 